\title{
Psoas Hematoma and Late Femoral Nerve Palsy After Extreme Lateral Interbody Fusion and Posterior Spinal Fusion with Instrumentation: A Case Report
}

This article was published in the following Dove Press journal:

Orthopedic Research and Reviews

\author{
Ibrahim S Almazrua (1D) \\ Abdullah Y Almarshad' \\ Ghadah Binzuman ${ }^{2}$ \\ Anwar M Alrabiah' \\ 'Department of Orthopedic Surgery, \\ King Faisal Specialist Hospital \& Research \\ Center, Riyadh, Saudi Arabia; ${ }^{2}$ College of \\ Medicine, Alfaisal University, Riyadh, \\ Saudi Arabia
}

\begin{abstract}
Introduction: Psoas hematoma is an uncommon complication following spinal surgeries. It has been reported in both extreme lateral interbody fusion (XLIF) and posterior spinal fusion with instrumentation. Minimally invasive techniques are gaining popularity in recent years due to the appealing advantages of reduced operative time, blood loss, hospital stay, and faster recovery.

Case Presentation: We are presenting a case of a 77-year-old male with chronic low back pain, diagnosed to have multilevel degenerative disc disease with central and foraminal disc protrusion at L2-L3, L3-L4, L4-L5 with secondary spinal stenosis, underwent XLIF at L3L4, L4-L5 and then 2nd stage with posterior L3-L5 fusion with pedicle screws. On the fourth day post-operatively, the patient had flank pain and dropping hemoglobin with femoral nerve palsy symptoms, a CT scan revealed a large psoas hematoma. Conservative management was decided on; a follow-up CT scan and examination showed complete resolution of the hematoma and femoral nerve recovery.
\end{abstract}

Discussion: The approach to iliopsoas hematoma post spinal surgeries remains controversial. Iliopsoas hematoma should be suspected in any patients post spinal surgeries even with delayed presentations. The decision to proceed with either surgical intervention or conservative management depends on multiple factors, including patient hemodynamic status, progression of collection and femoral nerve palsy.

Conclusion: The exact cause of iliopsoas hematoma post different spinal surgery approaches remains vague. In our opinion, other causes including pre- and post-operative anticoagulants should be investigated. Rushing to drain iliopsoas hematomas in case of femoral nerve palsy might not be the ideal option. Instead, monitoring patient responses to resuscitation and taking a watch and wait approach for femoral nerve palsy might be the proper approach.

Keywords: psoas hematoma, extreme lateral interbody fusion; XLIF, posterior spinal fusion, femoral nerve palsy

\section{Introduction}

Intramuscular psoas Hematoma is an uncommon condition and it has multiple causes including trauma, use of anticoagulation, iatrogenic injury during lumber procedure and bleeding disorders. ${ }^{1}$ Extreme Lateral Interbody Fusion (XLIF) is a far lateral approach; allowing access to the anterior aspect of the spine from the level of T6 to the level of L5. ${ }^{2}$ This minimally invasive surgery is retroperitoneal
Correspondence: Ibrahim S Almazrua Department of Orthopedic Surgery, King Faisal Specialist Hospital \& Research Center, P.O. Box 3354, Riyadh II2II Kingdom of Saudi Arabia

Tel +966 II 2162919 Ext. 46930

Email ibrahimalmazrua@hotmail.com
Orthopedic Research and Reviews 2020:12 127-132 
using a muscle splitting approach through the psoas muscle. ${ }^{2,3}$ Using this approach allows the surgeon to do a complete discectomy, distraction, and interbody fusion. ${ }^{3}$ Unlike anterior and posterior approaches to the spine, lateral approach is associated with less complications and morbidity rates. ${ }^{4}$ In addition, it has many advantages including reduced operative time, reduced blood loss, minimal wounds, shorter hospital stay and faster recovery time post-operatively. ${ }^{4}$ This can achieve reduction in the peri-operative morbidity through less invasive techniques and shorter hospitalization in order to address different spinal pathologies. ${ }^{5}$ Spinal canal stenosis is a pathological condition and it can be central, lateral recess or foraminal. Central and lateral stenosis can be decompressed using XLIF. ${ }^{6}$ Patient selection has very high importance in achieving satisfactory clinical outcomes to the patient and surgeon. For example, in anterior or posterior lumber interbody fusion approaches (ALIF, PLIF), the need for resection of important structures like anterior longitudinal ligament, posterior longitudinal ligament, facet joints, pars interarticularis and sometimes they are healthy structures, consequently need stabilization by instrumentation. Alternatively, XLIF respecting these structures and keeping them in tension in order to provide better stability and avoid instrumenting the spinal segments. ${ }^{7}$ However, combining XLIF and PLIF can be of great importance in providing extra stability in case of adult degenerative scoliosis. ${ }^{8}$ We report a case of large intramuscular iliopsoas hematoma post XLIF and PLIF for degenerative lumber scoliosis, presenting 4 days postoperatively with femoral nerve palsy. Treatment was conservative management with full recovery at 4 month follow-up. Written informed consent was obtained from the patient for publishing this paper.

\section{Case Presentation}

The patient is a 77-year-old male, with known cases of Rheumatoid Arthritis, Bronchial Asthma, and Chronic Obstructive Pulmonary disease. He presented to our clinic with a history of low back pain radiating to left lower extremity for several years. The pain was gradually increasing in severity, aggravated by walking up hill and relieved partially by pain medications associated with neurological claudications. There was no history of bowel bladder dysfunction or other cauda equina symptoms. The main complaint was pain that affected his daily living activities. Back examination revealed no obvious deformity with scar in the lumber area from previous surgery. No tenderness or hotness was appreciated. Flexion of 70 degree and extension of 20 degrees was achievable. The Adams forward bending test negative. A straight leg rise test was positive at 40 degrees hip flexion. Distal neurovascular exam was normal apart from decreased power on the left lower limb (4/5). The patient presented 2 years before the current presentation with a similar history of pain. An MRI at that time showed L4-L5 broad based central and right paracentral disc herniation with sever critical canal stenosis with foraminal narrowing related to disc hight loss and multilevel degenerative disc disease. For which he underwent L4-L5 lumber laminectomy and decompression. Post-operative period was unremarkable and patient discharged home. Pre-operative X-rays during this presentation showing significant instability and degenerative scoliosis is demonstrated in (Figure 1). MRI entire spine showed multilevel degenerative disc disease with L1-L2 and L2-L3 dorsal complex of disc protrusion and osteophytes, L3-L4 interval progression with secondary spinal stenosis, L4-L5 reduced spinal stenosis compared to previous images (Figure 2). Underwent XLIF technique at L3-L4, L4-L5 and then 2 nd stage with posterior L3-L5 fusion with pedicle screws with standard surgical steps without any complications (Figure 3). Four days post-operatively; the patient demonstrated lateral flank bruises and discoloration with dropping hemoglobin from 12 to $84 \mathrm{~g} / \mathrm{L}$ during follow-up complete blood count and left groin pain and numbness. An abdominal CT scan was obtained, showing large left-sided retroperitoneal hematoma measuring about $8.3 \times 8.5 \times 17 \mathrm{~cm}$ in $\mathrm{AP}$, transverse, and craniocaudal diameter respectively, inseparable from the left iliopsoas muscle (Figure 4). Moreover, post-operatively the patient was on heparin 5,000 units subcutaneously twice a day and his coagulation profile pre-operatively was normal, postoperatively was slightly disturbed with INR 1.3, PT 15.9 and PTT 38.5. Patient was not known to have any coagulopathy but he was on aspirin preoperatively, which was supposedly stopped 7 days before surgery, but the patient did not. General surgery and medical teams were involved, transfusion of 4 units of Packed Red Blood Cells (PRBC), 6 units of Fresh Frozen Plasma (FFP) and 2 units of platelets done for the patient. Patient was stable afterwards, and was on close observation. Conservative management was undertaken, with follow-up CT scan showing no progression. Patient was discharged few days later in stable condition with symptoms and signs of femoral nerve palsy; follow-up CT scans revealed resolution of the 


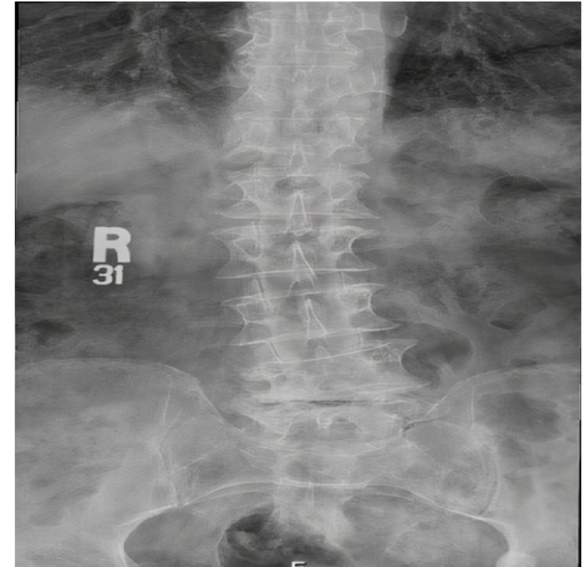

A

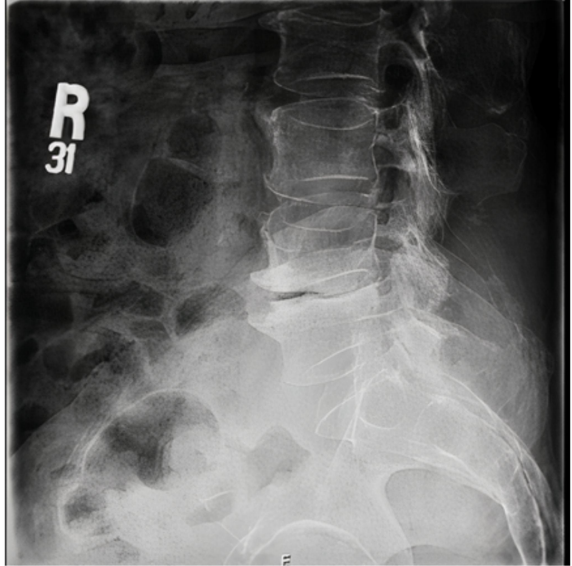

B

Figure I Pre-operative Anterioposterior (A) and Lateral (B) X-ray of lumbar spine.

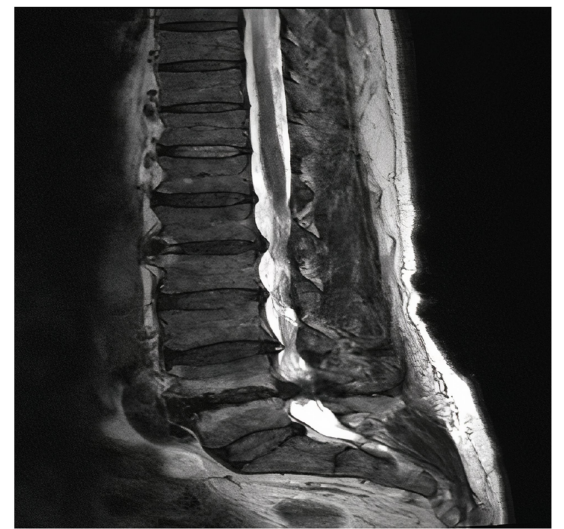

A

Figure 2 Pre-operative MRI of lumbar spine. (A) sagittal, (B) axial Level L4-L5.

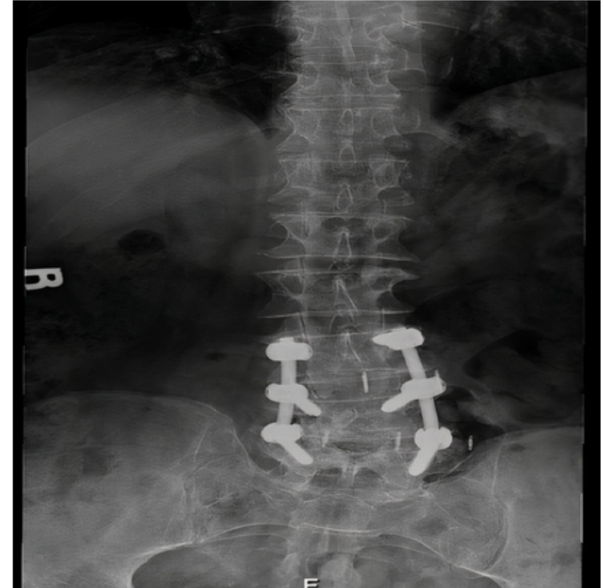

A

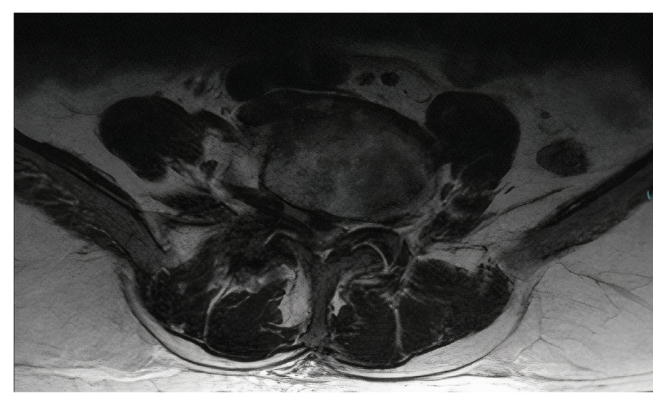

B

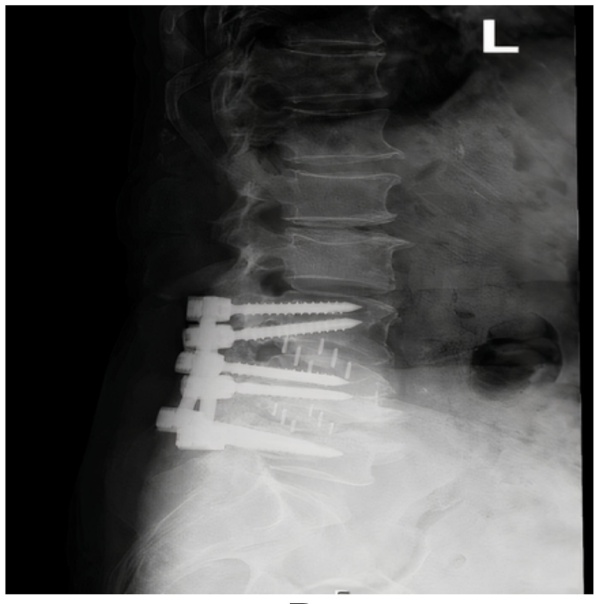

B

Figure 3 Post-operative Anterioposterior (A) and Lateral (B) X-rays of lumbar spine. 


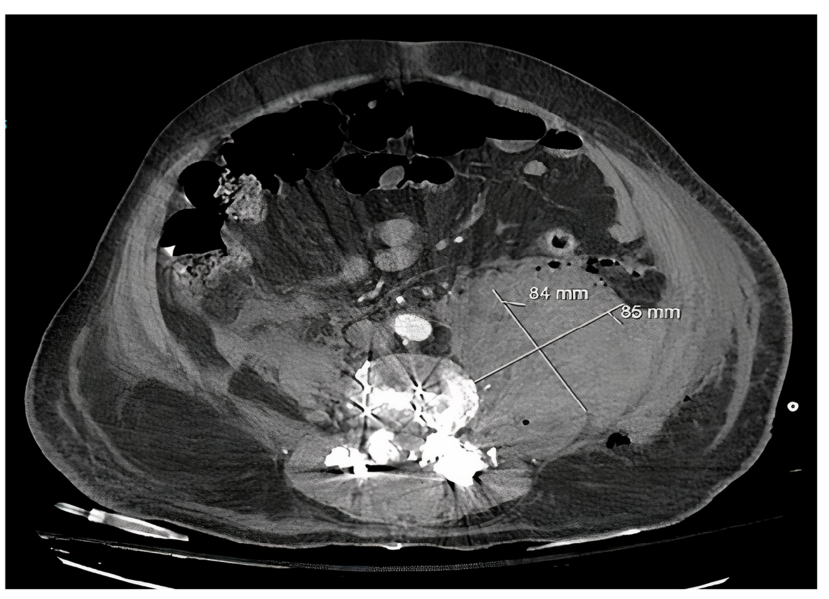

A

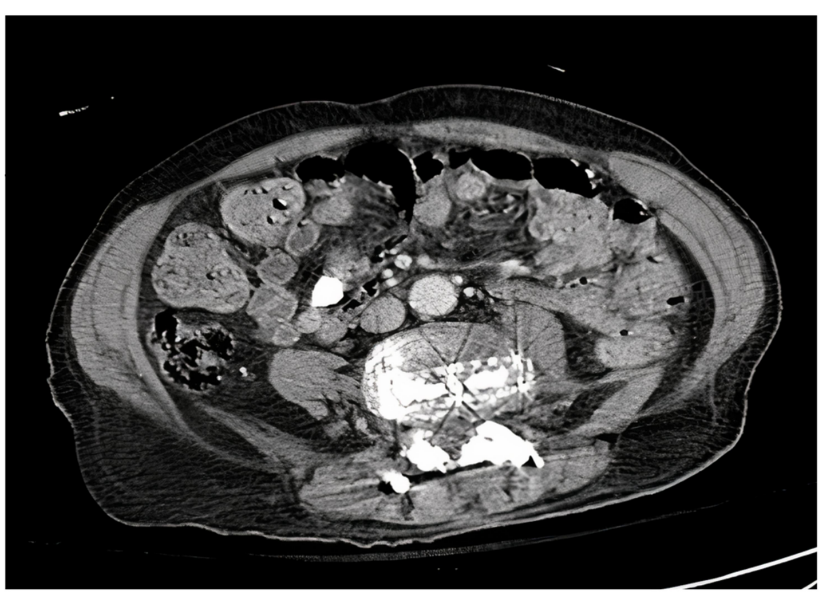

B

Figure 4 Post-operative CT scan showing psoas hematoma (A). Follow-up CT scan at 4 months showing complete resolution of psoas hematoma (B).

hematoma (Figure 4). Outpatient follow-up 4 months later revealed complete resolution of the palsy.

\section{Discussion}

XLIF is a minimally invasive technique, gaining popularity in recent years. Traditionally, interbody fusion is achieved through open surgical approach either through ALIF, PLIF or transforaminal interbody fusion (TLIF). ${ }^{9-}$ 12 Ozgur et al (2006) described XLIF thoroughly. With many advantages over other approaches including decreased risk of injury to great vessels, less operative time, less blood loss and eliminates the need for general surgery. ${ }^{3}$

Psoas hematoma presentation can be equivocal, leading to difficult and delayed diagnosis due to its retrofascial location. ${ }^{13}$ As in our case, 4 days later the patient started to have ecchymosis over the flank area with dropping hemoglobin post-operatively from 12 to 10 then $84 \mathrm{~g} / \mathrm{L}$ and hypotension with femoral nerve palsy. Despite that, there was no any active bleeding encountered during surgery and no immediate post-operative findings suggesting retroperitoneal hematoma. Varieties of symptoms were described for psoas hematoma including hip pain with the hip flexion position to decrease irritation to the psoas. Others may have radicular pain in the thigh due to compression of lumbar nerve roots, ${ }^{14}$ or femoral nerve palsy leading to weakness in hip flexion knee extension. ${ }^{15,16}$ Moreover, a fever should raise the suspicion of infection, including hematoma with superimposed infection with late presentation. However, it has been reported that hematoma may cause fever by the release of endogenous pyrogen or interleukin-1. ${ }^{17}$

A variety of etiologies for iliopsoas hematoma is described in the literature, including trauma, iatrogenic injury, use of anticoagulants, and coagulation disorders such as hemophilia and disseminated intravascular coagulopathy. ${ }^{14,18-22}$ However, our patient had a normal coagulation profile and no active bleeding during surgery. But he was on prophylactic heparin 5,000 units twice daily post-operatively and was on aspirin pre-operatively. Joshua et al reviewed 3,950 cases of minimally invasive lateral lumber retroperitoneal transpsoas interbody fusion, found 7 cases of iliopsoas hematoma $(0.18 \%), 3$ of which required surgical evacuation without improvement in neurological outcomes post-surgery. They documented only 1 case of confirmed segmental artery injury, while only suspected it in the rest of the patients. Moreover, only 2 patients were receiving anti-platelets prior to surgery. ${ }^{16,20}$ In addition, a retrospective study included 2,998 patients who underwent minimally invasive lateral interbody fusion reported a total of 5 cases of retroperitoneal hematoma. Three of which were identified to be due to segmental vessel injury. One patient was managed conservatively, while the others required intervention. ${ }^{23}$ Furthermore, others discussed the possibility of intertransverse plane injury in the development of iliopsoas hematoma. As described by Chung et al in a 13-year-old patient with adolescent idiopathic scoliosis required posterior spinal fusion and instrumentation from T11 to L4. During their dissection on the posterolateral gutter on the concave side of lumbar spine, fascia was violated at L2 level during exposure of the intertransverse plane. But no active bleeding was encountered and no neurological deficit was found post-operatively. They thought that it might be the reason behind iliopsoas hematoma. ${ }^{24,25}$ In general, the rate of iliopsoas hematoma 
post minimally invasive spine surgery is low. ${ }^{26,27}$ In

a review article discussing the complications associated with minimally invasive lateral interbody fusion, comparing prepsoas with transpsoas approaches, the authors found that the incidence rate of hematoma to be $1.5 \%$ (in 1,398 patients) and $1.7 \%$ (in 1,198 patients) respectively, without statistical significance. $^{27}$

The balance between bleeding and administration of anticoagulation therapy is challenging during perioperative period, especially in older patients with multiple co-morbidities. The risk of development of iliopsoas hematoma after anticoagulation therapy has been discussed by multiple authors. ${ }^{1,28,29}$ Sarwat et al reported a case of a 31-year-old female with intractable lower back pain, who underwent a dynamic stabilization procedure from L3 to L5. Post-operatively she received prophylactic anticoagulants. On the 3rd post-operative day she developed left groin pain and numbness in the thigh without motor deficit. MRI showed iliopsoas hematoma, for which they decided drainage under CT guidance. ${ }^{30}$ Others found that the incidence of spontaneous iliopsoas hematoma to be 3.8 cases per 1,000 admissions to intensive care units. They included 40 patients on either prophylactic or therapeutic anticoagulation, with more than half of them required embolization. ${ }^{28}$ In our case, the patient had no coagulopathy and no active bleeding during surgery. Our assumptions for the hematoma could be either due to postoperative prophylactic anticoagulation or pre-operative use of anti-platelets. Up to our knowledge, very few papers discussed the possible effect of pre-operative anti-platelets on the risk of development of iliopsoas hematoma.

The decision to drain or observe iliopsoas hematoma remains controversial. Despite the large collection in our case, conservative management with close observation was undertaken. Most of iliopsoas hematomas are managed conservatively if there was no clinical and hemodynamic deterioration. ${ }^{1,31}$ This can include stopping anticoagulants, fluid and blood administration and close observation with monitoring of hemoglobin and coagulation profile. ${ }^{32}$ However, surgical intervention is recommended by others in case of hemodynamic instability despite resuscitation and neurological deficit. ${ }^{33}$ Our patient had stable vital signs and hemoglobin level without coagulopathy post-blood transfusion. These factors guided conservative management together with the decisions of the primary team, general surgery team and medical teams to proceed with conservative management.

\section{Conclusion}

Iliopsoas hematoma following spinal surgery is considered a rare complication. Other causes should be investigated including post-operative anticoagulation and pre-operative use of antiplatelets therapy. Conservative management should be considered in case of stable hemodynamic patients with non progressive collection. Complete resolution of iliopsoas hematoma in our case on follow-up CT scans and femoral nerve palsy postulate that, even in cases of large hematomas, conservative management should be considered.

\section{Ethical Approval}

The study was approved by institutional review board by King Faisal Specialist Hospital \& Research Center.

\section{Acknowledgment}

The author would like to thank Dr. Omar A. Al-Mohrej for his support.

\section{Consent}

Written informed consent was obtained from the patient for publication of this case report and accompanying images.

\section{Disclosure}

The authors report no conflicts of interest for this work.

\section{References}

1. Seo JG, Yang JC, Kim TW, Park KH. Intramuscular hematoma on the psoas muscle. Korean J Neurotrauma. 2019;15(2):234-238. doi:10. 13004/kjnt.2019.15.e29

2. Berjano P, Lamartina C. Far lateral approaches (XLIF) in adult scoliosis. Eur Spine J. 2013;22(SUPPL.2):242-253. doi:10.1007/ s00586-012-2426-5

3. Ozgur BM, Aryan HE, Pimenta L, Taylor WR. Extreme Lateral Interbody Fusion (XLIF): a novel surgical technique for anterior lumbar interbody fusion. Spine J. 2006;6(4):435-443. doi:10.1016/j. spinee.2005.08.012

4. Billinghurst J, Akbarnia BA. Extreme lateral interbody fusion - XLIF. Curr Orthop Pract. 2009;20(3):238-251. doi:10.1097/BCO.0b013e3 181a32ead

5. Isaacs RE, Hyde J, Goodrich JA, Rodgers WB, Phillips FM. A prospective, nonrandomized, multicenter evaluation of extreme lateral interbody fusion for the treatment of adult degenerative scoliosis: perioperative outcomes and complications. Spine (Phila Pa 1976). 2010;35(SUPPL. 26S):322-330. doi:10.1097/BRS.0b013e3182022e04

6. Oliveira L, Marchi L, Coutinho E, Pimenta L. A radiographic assessment of the ability of the extreme lateral interbody fusion procedure to indirectly decompress the neural elements. Spine (Phila Pa 1976). 2010;35(SUPPL. 26S):331-337. doi:10.1097/BRS.0b013e3182022db0

7. Malham GM, Ellis NJ, Parker RM, et al. Maintenance of segmental lordosis and disk height in stand-alone and instrumented extreme lateral interbody fusion (XLIF). Clin Spine Surg. 2017;30(2):E90E98. doi:10.1097/BSD.0b013e3182aa4c94 
8. Tormenti MJ, Maserati MB, Bonfield CM, Okonkwo DO, Kanter AS. Complications and radiographic correction in adult scoliosis following combined transpsoas extreme lateral interbody fusion and posterior pedicle screw instrumentation. Neurosurg Focus. 2010;28(3):1-7. doi:10.3171/2010.1.FOCUS09263

9. Brau SA, Delamarter RB, Schiffman ML, Williams LA, Watkins RG. Vascular injury during anterior lumbar surgery. Spine J. 2004;4 (4):409-412. doi:10.1016/j.spinee.2003.12.003

10. Fantini GA, Pappou IP, Girardi FP, Sandhu HS, Cammisa FP. Major vascular injury during anterior lumbar spinal surgery: incidence, risk factors, and management. Spine (Phila Pa 1976). 2007;32 (24):2751-2758. doi:10.1097/BRS.0b013e31815a996e

11. DiPaola CP, Molinari RW. Posterior lumbar interbody fusion. $J \mathrm{Am}$ Acad Orthop Surg. 2008;16(3):130-139. doi:10.5435/00124635200803000-00004

12. Dhall SS, Wang MY, Mummaneni PV. Clinical and radiographic comparison of mini-open transforaminal lumbar interbody fusion with open transforaminal lumbar interbody fusion in 42 patients with long-term follow-up: clinical article. J Neurosurg Spine. 2008;9(6):560-565. doi:10.3171/SPI.2008.9.08142

13. Cronin CG, Lohan DG, Meehan CP, et al. Anatomy, pathology, imaging and intervention of the iliopsoas muscle revisited. Emerg Radiol. 2008;15(5):295-310. doi:10.1007/s10140-008-0703-8

14. Lakkol S, Sarda P, Karpe P, Krishna M. Conservative management of psoas haematoma following complex lumbar surgery. Indian J Orthop. 2014;48(1):107-110. doi:10.4103/0019-5413.125534

15. Ho KJ, Gawley SD, Young MR. Psoas haematoma and femoral neuropathy associated with enoxaparin therapy. Int $J$ Clin Pr. 2003;57(6):553-554.

16. Beckman JM, Vincent B, Park MS, et al. Contralateral psoas hematoma after minimally invasive, lateral retroperitoneal transpsoas lumbar interbody fusion: a multicenter review of 3950 lumbar levels. J Neurosurg Spine. 2016;26(1):50-54. doi:10.3171/2016.4.SPINE151040.

17. Chmel H, Palmer JA, Eikman EA. Soft tissue hematoma as a cause of fever in the adult. Diagn Microbiol Infect Dis. 1988;11(4):215-219. doi:10.1016/0732-8893(88)90007-7.

18. Margulies DR, Teng FW. Psoas muscle hematoma from blunt trauma: an unusual cause of severe abdominal pain. J Trauma. 1998;45 (1):155-156. doi:10.1097/00005373-199807000-00033

19. Basheer A, Jain R, Anton T, Rock J. Bilateral iliopsoas hematoma: case report and literature review. Surg Neurol Int. 2013. doi:10.4103/ 2152-7806.118561

20. Kim H, Ju C, Kim S, Kim J. Huge psoas muscle hematoma due to lumbar segmental vessel injury following percutaneous endoscopic lumbar discectomy. J Korean Neurosurg Soc. 2009;45(3):192-195. doi:10.3340/jkns.2009.45.3.192

21. Fernandez-paluzzi F, Hernandez SR, De Bosch NB, De Saez AR. Hematomas within the iliopsoas muscles in hemophilic patients. Clin Orthop Relat Res. 1996;328(328):19-24. doi:10.1097/00003086199607000-00005
22. Nezu K, Yoshioka T, Katayama H, Fukushi T, Kyan A. Spontaneous iliopsoas muscle hematoma secondary to disseminated intravascular coagulation caused by nafamostat mesilate allergy: a case study. Ren Replace Ther. 2019;5:1-6. doi:10.1186/s41100-019-0207-x

23. Fujibayashi S, Kawakami N, Asazuma T, et al. Complications associated with lateral interbody fusion: nationwide survey of 2998 cases during the first two years of its use in Japan. Spine (Phila Pa 1976). 2017;42(19):1478-1484. doi:10.1097/brs.0000000000002139

24. Chung MMTC, Cheung JPY. Psoas hematoma formation after violation of the intertransverse plane during posterior spinal surgery for adolescent idiopathic scoliosis. Acta Orthop Traumatol Turc. 2018;7-11. doi:10.1016/j.aott.2018.06.007

25. Robinson DE, Ball KE, Webb PJ. Iliopsoas hematoma with femoral neuropathy presenting a diagnostic dilemma after spinal decompression. Spine J. 2001;26(6):135-138. doi:10.1097/00007 632-200103150-00006

26. Zeng ZY, Xu ZW, He DW, et al. Complications and prevention strategies of oblique lateral interbody fusion technique. Orthop Surg. 2018;10(2):98-106. doi:10.1111/os. 12380

27. Walker CT, Harrison Farber S, Cole TS, et al. Complications for minimally invasive lateral interbody arthrodesis: a systematic review and meta-analysis comparing prepsoas and transpsoas approaches. J Neurosurg Spine. 2019;30(4):446-460. doi:10.3171/2018.9.SPINE 18800

28. Artzner T, Clere-jehl R, Schenck M, et al. Spontaneous ilio-psoas hematomas complicating intensive care unit hospitalizations. PLoS One. 2019:1-12. doi:10.1371/journal.pone.0211680.

29. Kim SW, Kim DH, Jung S. Spontaneous iliopsoas hematoma: a rare complication of anticoagulant use. Arch Plast Surg. 2015;42 (4):507-510. doi:10.5999/aps.2015.42.4.507

30. Sarwat AM, Sutcliffe JC. Haematoma of the psoas muscle after posterior spinal instrumentation and Enoxaparin prophylaxis. $G d$ Rounds. 2003;3:35-37. doi:10.1102/1470-5206.2003.0012

31. Retselas IN, Vaiopoulos CK, Chronopoulos PN, Kaisidis GN, Fotopoulou CE, Skandalos IK. Spontaneous iliopsoas muscle haematoma during anticoagulant treatment. Hell $J$ Surg. 2015;87 (5):434-436. doi:10.1007/s13126-015-0254-x

32. Ozkan OF, Guner A, Cekic AB, Turan T, Kaya U, Reis E. Iliopsoas haematoma: a rare complication of warfarin therapy. J Coll Physicians Surg Pak. 2012;22(10):673-674. doi:10.2012/JCPSP.673674

33. Parmer SS, Carpenter JP, Fairman RM, Velazquez OC, Mitchell ME. Femoral neuropathy following retroperitoneal hemorrhage: case series and review of the literature. Ann Vasc Surg. 2006;20(4):536-540. doi:10.1007/s10016-006-9059-2
Orthopedic Research and Reviews

\section{Publish your work in this journal}

Orthopedic Research and Reviews is an international, peer-reviewed, open access journal that focusing on the patho-physiology of the musculoskeletal system, trauma, surgery and other corrective interventions to restore mobility and function. Advances in new technologies, materials, techniques and pharmacological agents are particularly welcome. The manuscript management system is completely online and includes a very quick and fair peer-review system, which is all easy to use. Visit http://www.dovepress.com/testimonials.php to read real quotes from published authors. 